\title{
The Role of the Cell Adhesion Molecules (integrins / cadherins) in Prostate Cancer
}

\author{
Alexandros Drivalos, Athanasios G. Papatsoris, Michael Chrisofos, Eleni Efstathiou, Meletios \\ A. Dimopoulos
}

2nd Department of Urology (AD, AGP, MC), School of Medicine, University of Athens, Sismanoglio Hospital, Athens, Greece and Department of Clinical Therapeutics / Oncology (EE, MAD), School of Medicine, University of Athens, Alexandra Hospital, Athens, Greece

\begin{abstract}
During prostate carcinogenesis the cellular adhesion molecules, i.e.; integrins and cadherins mediate aberrant interactions between glandular epithelial cells and the extracellular matrix. Several integrin $\alpha$ subunits are downregulated, while $\beta$ subunits are up-regulated. The expression of several cadherins and catenins has specific prognostic value. There is an association between the expression of the E-cadherin/catenin complex and high grade prostate cancer. Clinical trials evaluating the efficacy of integrin antagonists are ongoing with promising results. In this article we update the role of integrins and cadherins in prostate carcinogenesis and evaluate the therapeutic potential of their manipulation.
\end{abstract}

Key words: cadherins; integrins; prostate cancer; review

Int Braz J Urol. 2011; 37: 302-306

\section{INTRODUCTION}

During prostate cancer (PCa) progression, precursor lesions will eventually progress to incurable androgen-independent metastatic disease (1). Among the alterations described in prostate carcinogenesis are aberrant interactions between glandular epithelial cells and the extracellular matrix (ECM), mediated by cell adhesion molecules (CAMs). CAMs are divided into four major categories: cadherins, integrins, selectins and members of the immunoglobulin superfamily. The majority of studies focus on the abnormal expression of cadherins and integrins. In this article, we update current knowledge and their role in prostate carcinogenesis and evaluate the therapeutic potential of manipulating these molecules.

\section{ROLE OF INTEGRINS AND CADHERINS}

\section{Integrins}

Integrins are transmembrane glycoprotein receptors for ECM proteins. They are composed of two subunits $\alpha$ and $\beta$, the combination of which gives them a different specificity and function. Currently, 26 members of the family of integrins have been described (18 $\alpha$ and $8 \beta$ subunits) (2). The extracellular domain of integrins has binding sites and upon interaction with the ECM, they form links between cells and ECM components.

\section{Cadherins}

Cadherins are transmembrane glycoproteins that mediate intercellular adhesion in the 
presence of extracellular calcium (3). Cell-cell adhesion in cadherins is mainly mediated by homotypic interactions. There are about 20 different cadherin molecules currently classified and the most extensively studied are: E- (epithelial), N- (neural) and P- (placental) cadherins. The cadherin complex is composed of the following components: cadherin, intracellular components associated with cadherin (i.e. catenins), and the cytoskeleton (i.e. actin). Catenins (main types are: $\alpha-, \beta-$ and $\gamma-$ ) are cytoplasmic proteins that interact with the intracellular domain of cadherins, providing anchorage to the microfilament cytoskeleton.

\section{EXPRESSION OF INTEGRINS IN PCA}

\section{a subunit}

High Gleason score has been correlated with low and/or negative expression of integrin subunit $\alpha_{3}$ (4). The expression of $\alpha 6$ subunit diminishes with increasing histologic grade, especially at sites of contact with the basement membrane (5). PCa demonstrates decreased positive staining for subunit $\alpha_{7}$, with a further decrease in metastatic disease (6). Other $\alpha$ subunits that have been found down-regulated include $\alpha_{2}, \alpha_{4}, \alpha_{5}$, and $\alpha \mathrm{v}$ (2). Interestingly, $\alpha_{\mathrm{II} \beta}$ subunit is expressed only in PCa and not in normal tissue (7).

\section{B subunit}

Five $\beta_{1}$ variant subunits have been described and two of them $\left(\beta_{1 \mathrm{C}}\right.$ and $\left.\beta_{1 \mathrm{~A}}\right)$ are expressed in normal prostatic epithelium. Decreased $\beta_{1 \mathrm{C}}$ steady-state mRNA levels were detected in $94 \%$ of PCa specimens compared with normal samples (8). Subunit $\beta_{1 \mathrm{~A}}$ has been found to be up-regulated in human $\mathrm{PCa}$, as well as, in TRAMP (transgenic adenocarcinoma of mouse prostate) models (9). The expression of $\beta 1 \mathrm{~A}$ promotes cell proliferation and survival by increasing the expression of insulin-like growth factor receptor type 1 (IGFR-1) (9).

Upregulation of $\beta_{3}$ and $\beta_{6}$ integrin subunits has been described in PCa. Studies have demonstrated expression of the heterodimeric complexes $\alpha v \beta_{3}$ and $\alpha v \beta_{6}$, in PCa specimens in contrast with normal controls (10). Integrin $\alpha_{v} \beta_{3}$ has been demonstrated to increase cdc2 kinase activity and cdc2 levels in PCA (11). Higher cdc2 levels result in increased cell migration mediated by interaction of cdc2 with cyclin B2 and phosphorylation of caldesmon. It is known that cdc2 and caldesmon are localized in the membrane ruffles of motile cells.

\section{EXPRESSION OF CADHERINS IN PCA}

\section{Cadherins}

It has been demonstrated that tumor tissues exhibit decreased levels of E-cadherin (12). The expression of this cadherin was significantly correlated with histological differentiation and bones metastasis, but not with lymphatic or vascular invasion (13). Several studies have demonstrated an association between the expression of the E-cadherin/catenin complex and high grade PCa (14). Richmond et al. (15) demonstrated that abnormal expression of the E-cadherin/ $\alpha$-catenin complex was significantly correlated with Gleason score and with a lower survival outcome. In a recent study it has been demonstrated that there is a significant decrease of membrane expression of E-cadherin/ $\beta$-catenin complex and an increase of cytoplasmatic and nuclear location of the same complex, in high Gleason score prostate cancer (16). A low E-cadherin to high N-cadherin expression switch has been correlated with progression of $\mathrm{PCa}$ and high mortality. N-cadherin is not expressed in normal prostate tissue; however, in PCa it has been detected especially in poorly differentiated areas (17). Also, in a number of metastatic lesions, $\mathrm{N}$-cadherin has been found to be homogeneously expressed.

Prostatic tumor tissues were demonstrated to exhibit decreased levels of P-cadherin (12) even when compared with the reduced expression in benign prostatic hyperplasia (BPH). Furthermore, Cadherin-11 is expressed in the stroma of all $\mathrm{PCa}$ specimens. A recent study has show the absence of cadherin-10 in irregular cancer profiles in contrast with normal glandular profiles (18).

\section{Catenins}

Aberrant expression of all main three catenin types ( $\alpha, \beta$ - and $\gamma$-catenin) has been associated with 
extra prostatic extension (14). The expression of $\alpha$-catenin seems to have prognostic value in both local and locally advanced PCa $(14,15)$. The abnormality in $\alpha$-catenin expression has been found to be associated with high Gleason score, perineural invasion and poor survival. Furthermore, the expression of $\alpha$-catenin in PCa is up-regulated not only in comparison with normal tissue, but also when compared with the elevated expression in BPH. Hyperplastic prostates differ from normal prostates in the weaker immunoreactions of the two catenins $\beta$ - and $\gamma-$, as well as, in the intracellular distribution of them (19). A recent study has demonstrated that the membranous overexpression of $\beta$-catenin is significantly associated with the metastatic prostate cancer cells in the bone and that the high frequency of expression suggests its involvement in the intercellular adhesion of the metastatic cells in the bone. Furthermore, studies have demonstrated that $\delta$-catenin is overexpressed in $\mathrm{PCa}$ and is correlated positively with increasing Gleason scores (14).

\section{CLINICAL IMPLICATIONS}

Clinical trials that evaluate the efficacy of integrin antagonists as $\mathrm{PCa}$ therapeutics are ongoing. Cilengitide, a cyclic Arg-Gly-Asp peptide that inhibits $\alpha v \beta_{3}$ and $\alpha v \beta_{5}$, is a promising antagonist of $\alpha \mathrm{v}$ integrins. A recent randomized phase II trial assessed the efficacy of different doses of Cilengitide in metastatic hormone refractory PCa patients (20). Forty-four patients were randomized to Cilengitide $500 \mathrm{mg}$ or 2,000 mg IV twice weekly. Median number of cycles was three in both arms and at 6 months two patients on the $500 \mathrm{mg}$ arm and five on the 2,000 $\mathrm{mg}$ arm had not progressed, while there was no grade 4 toxicity.

Monoclonal antibodies that inhibit $\alpha \mathrm{v}$ integrins have also been studied, usually in conjunction with standard chemotherapy. CNTO 95, an $\alpha$ v integrin monoclonal antibody, was administered $(10 \mathrm{mg} /$ $\mathrm{kg}$, once weekly) in combination with docetaxel and prednisone and appeared to be well tolerated (21). MEDI-522 is an antibody against the $\alpha_{\mathrm{v}} \beta_{3}$ integrin that is being studied in a randomized phase II in combination with docetaxel, prednisone and zoledronic acid [unpublished data].
It is questionable if E-cadherin, $\beta$-catenin or $\alpha$-catenin, can be used individually as prognostic markers. However, a combination of these proteins may be of clinical use as in other tumors (i.e. liver, colorectal). Apigenin is a plant flavone that blocks $\beta$-catenin signaling and has been found to suppress prostate carcinogenesis in TRAMP mice (22). Another study (23) has suggested that therapeutic strategies blocking cadherin-11 expression or function, such as anti-cadherin-11 antibodies, may be considered when applying androgen-ablation therapy, because androgen deprivation up-regulates cadherin-11 expression in $\mathrm{PCa}$, and this may contribute to the metastasis of PCa. Finally, a study (24) examined the potential of an N-cadherin inhibitor: ADH1, in reducing tumor angiogenesis ex vivo and delaying tumor progression in vivo. Relevant results have demonstrated that $\mathrm{ADH} 1$, does not display antiangiogenic activity in a rat aortic ring assay or antitumor potential in a PC3 subcutaneous xenograft tumor model. Cytotoxic activity was detected in human umbilical vein endothelial cells, PC3, and Tsu-Pr1 cells.

\section{CONCLUSIONS}

The molecular pathways by which integrins and cadherins contribute to PCa progression need to be further elucidated. The expression of several cadherins and catenins has specific prognostic value that could be standardized. Finally, designing novel therapeutic approaches based on inhibiting integrin expression and/or downstream signaling is likely to be a promising strategy.

\section{CONFLICT OF INTEREST}

None declared.

\section{REFERENCES}

1. Chrisofos M, Papatsoris AG, Lazaris A, Deliveliotis C: Precursor lesions of prostate cancer. Crit Rev Clin Lab Sci. 2007; 44: 243-70.

2. Ramsay AG, Marshall JF, Hart IR: Integrin trafficking and its role in cancer metastasis. Cancer 
Metastasis Rev. 2007; 26: 567-78.

3. Halbleib JM, Nelson WJ: Cadherins in development: cell adhesion, sorting, and tissue morphogenesis. Genes Dev. 2006; 20: 3199-214.

4. Schmelz M, Cress AE, Scott KM, Bürger F, Cui $\mathrm{H}$, Sallam K, et al.: Different phenotypes in human prostate cancer: alpha6 or alpha3 integrin in cell-extracellular adhesion sites. Neoplasia. 2002; 4: 243-54.

5. King TE, Pawar SC, Majuta L, Sroka IC, Wynn $\mathrm{D}$, Manolis C, et al.: The role of alpha 6 integrin in prostate cancer migration and bone pain in a novel xenograft model. PLoS One. 2008; 3:e3535.

6. Ren $\mathrm{B}, \mathrm{Yu} \mathrm{YP}$, Tseng GC, Wu C, Chen K, Rao $\mathrm{UN}$, et al.: Analysis of integrin alpha7 mutations in prostate cancer, liver cancer, glioblastoma multiforme, and leiomyosarcoma. J Natl Cancer Inst. 2007; 99: 868-80.

7. Trikha M, Cai Y, Grignon D, Honn KV: Identification of a novel truncated alphaIIb integrin. Cancer Res. 1998; 58: 4771-5.

8. Perlino E, Lovecchio M, Vacca RA, Fornaro M, Moro L, Ditonno P: Regulation of mRNA and protein levels of beta 1 integrin variants in human prostate carcinoma. Am J Pathol. 2000; 157: 172734.

9. Goel HL, Breen M, Zhang J, Das I, AznavoorianCheshire S, Greenberg NM, et al.: beta1A integrin expression is required for type 1 insulin-like growth factor receptor mitogenic and transforming activities and localization to focal contacts. Cancer Res. 2005; 65: 6692-700.

10. Zheng DQ, Woodard AS, Fornaro M, Tallini G, Languino LR: Prostatic carcinoma cell migration via alpha(v)beta3 integrin is modulated by a focal adhesion kinase pathway. Cancer Res. 1999; 59: 1655-64.

11. Manes T, Zheng DQ, Tognin S, Woodard AS, Marchisio PC, Languino LR: Alpha(v)beta3 integrin expression up-regulates cdc2, which modulates cell migration. J Cell Biol. 2003; 161: 817-26.

12. Wang J, Krill D, Torbenson M, Wang Q, Bisceglia M, Stoner J, et al.: Expression of cadherins and catenins in paired tumor and non-neoplastic primary prostate cultures and corresponding prostatectomy specimens. Urol Res. 2000; 28: 308-15.

13. Pontes J Jr, Srougi M, Borra PM, Dall' Oglio MF, Ribeiro-Filho LA, Leite KR: E-cadherin and betacatenin loss of expression related to bone metastasis in prostate cancer. Appl Immunohistochem Mol Morphol. 2010; 18: 179-84.
14. van Oort IM, Tomita K, van Bokhoven A, Bussemakers MJ, Kiemeney LA, Karthaus HF, et al.: The prognostic value of E-cadherin and the cadherin-associated molecules alpha-, beta-, gammacatenin and $\mathrm{p} 120 \mathrm{ctn}$ in prostate cancer specific survival: a long-term follow-up study. Prostate. 2007; 67: 1432-8.

15. Richmond PJ, Karayiannakis AJ, Nagafuchi A, Kaisary AV, Pignatelli M: Aberrant E-cadherin and alpha-catenin expression in prostate cancer: correlation with patient survival. Cancer Res. 1997; 57: 3189-93.

16. Aaltomaa S, Lipponen P, Ala-Opas M, Eskelinen M, Kosma VM: Alpha-catenin expression has prognostic value in local and locally advanced prostate cancer. Br J Cancer. 1999; 80: 477-82.

17. Contreras HR, Ledezma RA, Vergara J, Cifuentes F, Barra C, Cabello P, et al.: The expression of syndecan-1 and -2 is associated with Gleason score and epithelial-mesenchymal transition markers, E-cadherin and beta-catenin, in prostate cancer. Urol Oncol. 2010; 28: 534-40.

18. Walker MM, Ellis SM, Auza MJ, Patel A, Clark P: The intercellular adhesion molecule, cadherin-10, is a marker for human prostate luminal epithelial cells that is not expressed in prostate cancer. Mod Pathol. 2008; 21: 85-95.

19. Arenas MI, Romo E, Royuela M, Fraile B, Paniagua R: E-, N- and P-cadherin, and alpha-, betaand gamma-catenin protein expression in normal, hyperplastic and carcinomatous human prostate. Histochem J. 2000; 32: 659-67.

20. Bradley DA, Daignault S, Ryan CJ, Dipaola RS, Smith DC, Small E, et al.: Cilengitide (EMD 121974, NSC 707544) in asymptomatic metastatic castration resistant prostate cancer patients: a randomized phase II trial by the prostate cancer clinical trials consortium. Invest New Drugs. 2010; 25. [Epub ahead of print]

21. Chu FM, Picus J, Fracasso PM, Dreicer R, Lang Z, Foster B: A phase 1, multicenter, open-label study of the safety of two dose levels of a human monoclonal antibody to human alpha(v) integrins, intetumumab, in combination with docetaxel and prednisone in patients with castrate-resistant metastatic prostate cancer. Invest New Drugs. 2010; 11. [Epub ahead of print]

22. Shukla S, MacLennan GT, Flask CA, Fu P, Mishra A, Resnick MI, wt al.: Blockade of beta-catenin signaling by plant flavonoid apigenin suppresses prostate carcinogenesis in TRAMP mice. Cancer 
Res. 2007; 67: 6925-35.

23. Lee YC, Cheng CJ, Huang M, Bilen MA, Ye X, Navone NM, et al.: Androgen depletion up-regulates cadherin-11 expression in prostate cancer. J Pathol. 2010; 221: 68-76.

\section{Correspondence address:}

Dr. Athanasios G Papatsoris

Kanari 9, 10671, Athens, Greece

Fax: + 30210 804-4703

E-mail: agpapatsoris@yahoo.gr
24. Li H, Price DK, Figg WD: ADH1, an N-cadherin inhibitor, evaluated in preclinical models of angiogenesis and androgen-independent prostate cancer. Anticancer Drugs. 2007; 18: 563-8. 